PROCEEDINGS OF THE

AMERICAN MATHEMATICAL SOCIETY

Volume 126, Number 5, May 1998, Pages 1445-1451

S 0002-9939(98)04523-7

\title{
ON THE REDUCIBILITY \\ OF LINEAR DIFFERENTIAL EQUATIONS WITH QUASIPERIODIC COEFFICIENTS WHICH ARE DEGENERATE
}

\author{
XU JUNXIANG AND ZHENG QIN
}

(Communicated by Hal L. Smith)

\begin{abstract}
This paper proves the reducibility of a class of linear differential equations with quasiperiodic coefficients which are degenerate with respect to a small perturbation parameter. Our results generalize some that were obtained by Jorba and Simó.
\end{abstract}

\section{INTRODUCTION AND MAIN RESULTS}

Notation and definitions. The function $f(t)$ is called a quasiperiodic function of $t$ with frequencies $\omega_{1}, \omega_{2}, \cdots, \omega_{r}$, if there is a function $F\left(\theta_{1}, \theta_{2}, \cdots, \theta_{r}\right)$, which is $2 \pi$-periodic in all its arguments $\theta_{i}(i=1,2, \cdots, r)$, such that $f(t)=$ $F\left(\omega_{1} t, \omega_{2} t, \cdots, \omega_{r} t\right)$. If $F(\theta)=F\left(\theta_{1}, \theta_{2}, \cdots, \theta_{r}\right) \quad\left(\theta=\left(\theta_{1}, \theta_{2}, \cdots, \theta_{r}\right)\right)$ is analytic on a strip $D_{\rho}=\left\{\theta \in C^{r} \quad|| \operatorname{Im} \theta_{j} \mid \leq \rho, j=1,2, \cdots, r\right\}$, we say that $f(t)$ is analytic quasiperiodic in $D_{\rho}$. Denote the sup-norm of $f$ on $D_{\rho}$ by $\|f\|_{\rho}=\sup _{\theta \in D_{\rho}}|F(\theta)|$.

An $n \times n$ matrix $Q(t)=\left(q_{i j}(t)\right)_{1 \leq i, j \leq n}$ is called analytic quasiperiodic on $D_{\rho}$ with frequencies $\omega_{1}, \omega_{2}, \cdots, \omega_{r}$ if $q_{i j}(i, j=1,2, \cdots, n)$ are all analytic quasiperiodic on $D_{\rho}$ with the frequencies $\omega_{1}, \omega_{2}, \cdots, \omega_{r}$. Define a matrix norm of $Q$ by $\|Q\|_{\rho}=$ $n \times \max _{1 \leq i, j \leq n}\left\|q_{i j}\right\|_{\rho}$. It is easy to see that $\left\|Q_{1} Q_{2}\right\|_{\rho} \leq\left\|Q_{1}\right\|_{\rho} \cdot\left\|Q_{2}\right\|_{\rho}$. If $Q$ is a constant matrix, set $\|Q\|=\|Q\|_{\rho}$ for simplicity. Write the avarage of $Q(t)$ as $\bar{Q}=\left(\bar{q}_{i j}\right)_{1 \leq i, j \leq n}$, where $\bar{q}_{i j}=\lim _{T \rightarrow \infty} \frac{1}{2 T} \int_{-T}^{T} q_{i j}(t) d t$; for the existence of the limit, see [1]. Let $A(t)$ be an $n \times n$ quasiperiodic matrix. The differential equations $\dot{x}=A(t) x, x \in R^{n}$, are called reducible if there exists a nonsingular quasiperiodic change of variables $x=\Phi(t) y$ such that $\Phi(t)$ and $\Phi^{-1}(t)$ are quasiperiodic and bounded, and such that it changes the equations to $\dot{y}=B y$, where $B$ is a constant matrix.

Problems. In this paper we consider the reducibility of the following linear differential equations:

$$
\dot{x}=(A+\epsilon Q(t)) x, \quad x \in R^{n},
$$

Received by the editors October 22, 1996.

1991 Mathematics Subject Classification. Primary 34D20; Secondary 34C05.

Key words and phrases. Linear differential equations, reducibility, quasiperiodic, KAM iteration. 
where $A$ is an $n \times n$ constant matrix with different eigenvalues $\lambda_{1}, \lambda_{2}, \cdots, \lambda_{n}, Q(t)$ is an $n \times n$ quasiperiodic matrix of time $t$ with frequencies $\omega_{1}, \omega_{2}, \cdots, \omega_{r}$, and $\epsilon$ is a small perturbation parameter.

This problem was considered by Jorba and Simó in [2]. They proved that if

$$
\left|\langle\omega, k\rangle \sqrt{-1}+\lambda_{i}-\lambda_{j}\right| \geq \frac{\alpha}{|k|^{\tau}}, \forall 0 \neq k \in Z^{r}, \forall i, j=1,2, \cdots, n,
$$

and

$$
\left.\frac{d}{d \epsilon}\left(\bar{\lambda}_{i}(\epsilon)-\bar{\lambda}_{j}(\epsilon)\right)\right|_{\epsilon=0} \neq 0, \quad i \neq j
$$

where $\alpha>0$ and $\tau>r-1$ are constants and $\bar{\lambda}_{i}(\epsilon)(i=1,2, \cdots, n)$ are eigenvalues of $A+\epsilon \bar{Q}$, then, for sufficiently small $\epsilon_{0}>0$, there exists a nonempty Cantor subset $E \subset\left(0, \epsilon_{0}\right)$ such that for $\epsilon \in\left(0, \epsilon_{0}\right)$ the equations (1.1) are reducible.

The method used in [2] is a kind of KAM iteration. In the KAM iteration the difficulty is caused by a small divisor $\langle\omega, k\rangle+\lambda_{i}-\lambda_{j}$. The small divisor conditions (1.2) are necessary to overcome the difficulty of the small divisor. Since the frequencies $\omega$ are invariant in the iteration, which is different from the usual KAM iteration [4], one must adjust the small parameter $\epsilon \in\left(0, \epsilon_{0}\right)$ to guarantee the small divisor conditions. So Jorba and Simó needed the nondegeneracy conditions (1.3) to guarantee existence of the nonempty Cantor subset $E$, on which all the small divisor conditions in the KAM iterations hold.

If the nondegeneracy conditions (1.3) do not hold, we say $\bar{\lambda}_{i}(\epsilon)-\bar{\lambda}_{j}(\epsilon)$ are degenerate. This degenerate case is mentioned in [2], but there is no any result given. Like the motivation of [2], in this paper we want to consider this degenerate case. We will prove a similar result under weaker nondegeneracy conditions. Moreover, in the situation of the usual nondegeneracy conditions (1.3), our result is just the same as that of [2], but the proof is simpler.

\section{Main result.}

Theorem. Suppose $A=\operatorname{diag}\left(\lambda_{1}, \lambda_{2}, \cdots, \lambda_{n}\right)$ with $\lambda_{i} \neq \lambda_{j}$ for $i \neq j, 1 \leq i, j \leq n$, and $Q(t)=\left(q_{i j}(t)\right)_{1 \leq i, j \leq n}=\sum_{k \in Z^{r}} Q_{k} e^{i\langle k, \omega\rangle t}$ is an analytic quasiperiodic matrix on $D_{\rho}$ with frequencies $\omega_{1}, \omega_{2}, \cdots, \omega_{r}$, where the Fourier coefficients $Q_{k}$ depend analytically on the small parameter $\epsilon$. Let $Q_{0}^{d}=\operatorname{diag}\left(\bar{q}_{11}, \bar{q}_{22}, \cdots, \bar{q}_{n n}\right)$, where $\bar{q}_{i i}$ is the average of $q_{i i}(t)$. Suppose that for $i \neq j, \epsilon\left(\bar{q}_{i i}-\bar{q}_{j j}\right)$ has one of the following forms:

$$
\mu_{1} \epsilon^{l_{1}}+o\left(\epsilon^{l_{1}}\right), \mu_{2} \epsilon^{l_{2}}+o\left(\epsilon^{l_{2}}\right), \cdots, \mu_{p} \epsilon^{l_{p}}+o\left(\epsilon^{l_{p}}\right),
$$

where $\mu_{i} \neq 0, i=1,2, \cdots, p, 1 \leq l_{1}<l_{2}<\cdots<l_{p}$, and $o\left(\epsilon^{l}\right)$ is of order smaller than $\epsilon^{l}$ as $\epsilon \rightarrow 0$. Suppose

(1) (nonresonance conditions) $\lambda=\left(\lambda_{1}, \lambda_{2}, \cdots, \lambda_{n}\right)$ and $\omega=\left(\omega_{1}, \omega_{2}, \cdots, \omega_{r}\right)$ satisfy

$$
\left|\langle k, \omega\rangle+\lambda_{i}-\lambda_{j}\right| \geq \frac{\alpha}{|k|^{\tau}}, \forall 0 \neq k \in Z^{r}, \forall 1 \leq i, j \leq n,
$$

where $\alpha>0, \tau>r-1$. and

(2) $Q$ is $l_{p}$ order continuously differentiable with respect to sufficiently small $\epsilon$

$$
\left\|\frac{d^{l} \tilde{Q}}{d \epsilon^{l}}\right\|_{D_{\rho}} \leq M_{l}, l=0,1,2, \cdots, l_{p}
$$


where $\tilde{Q}=Q-Q_{0}^{d}$. Then, there exist $N_{1}, N_{2}, \cdots, N_{p}, N_{i}$ depending on $M_{1}, M_{2}, \cdots$, $M_{l_{j}}, \alpha, \tau, n, l_{p}$ and $\mu_{1} \epsilon^{l_{1}}+o\left(\epsilon^{l_{1}}\right), \cdots, \mu_{j} \epsilon^{l_{j}}+o\left(\epsilon^{l_{j}}\right)$, where $l_{j} \leq l_{i}-2$, such that if $\left|\mu_{i}\right|>N_{i}, i=1,2, \cdots, p$, then for sufficiently small $\epsilon_{0}>0$, there exists a nonempty Cantor subset $E \subset\left(0, \epsilon_{0}\right)$ with positive Lebesgue measure such that for $\epsilon \in E$ the equations (1.1) are reducible, i.e., there exists a nonsingular quasiperiodic transformation $x=\Phi(t) y$ that changes (1.1) to $\dot{y}=B y$, where $B$ is a constant matrix. If $\epsilon_{0}$ is small enough, the relative measure of $E$ in $\left(0, \epsilon_{0}\right)$ is close to 1. Moreover, the quasiperiodic matrix $\Phi(t)$ has the same frequencies as $Q(t)$.

Remark 1. If $l_{1}=1$, we can choose $N_{1}=0$. If $\epsilon\left(\bar{q}_{i i}-\bar{q}_{j j}\right)$ take only the form $\mu \epsilon+o(\epsilon)$, this corresponds to the nondegenerate case of [2].

Remark 2. There are many $\omega$ and $\lambda$ satisfying the nonresonance conditions in the theorem. We refer to [2], [3] for detailed discussions about nonresonance conditions or small divisor conditions.

\section{Proof of the main Result}

In this section we prove the theorem by the same idea of [2] with a little modification, which can simplify the proof in the nondegenerate case.

A. Outline of the proof. Write the equations (1.1) as

$$
\dot{x}=\left(A^{+}+\epsilon \tilde{Q}(t)\right) x,
$$

where $A^{+}=\operatorname{diag}\left(\lambda_{1}^{+}, \lambda_{2}^{+}, \cdots, \lambda_{n}^{+}\right)=A+\epsilon Q_{0}^{d}, \tilde{Q}=Q(t)-\epsilon Q_{0}^{d}$. From [2] we know that under the change of variables $x=(I+\epsilon P(t)) y$, where $P$ satisfies

$$
\dot{P}=A^{+} P-P A^{+}+\tilde{Q},
$$

the equations (2.1) are changed to

$$
\dot{y}=\left(A^{+}+\epsilon^{2} Q_{+}(t) P\right) y,
$$

where $Q_{+}=(I+\epsilon P)^{-1} \tilde{Q} P$. If the above process can go on, then the perturbation term $\epsilon^{2} Q_{+}(t)$ becomes smaller and smaller and the equations converge to constant coefficient equations.

The key to the iteration is to solve the equation (2.2) for $P$. Denote by $P=$ $\left(p_{i j}(t)\right)_{1 \leq i, j \leq n}, \quad \tilde{Q}=\left(\tilde{q}_{i j}(t)\right)_{1 \leq i, j \leq n}$. Expanding them in Fourier series and substituting them into the equations (2.2), by comparing the coefficients of both sides of the equations, we see formally that

If

$$
p_{i j}^{k}=\frac{\tilde{q}_{i j}^{k}}{\langle k, \omega\rangle \sqrt{-1}+\lambda_{i}^{+}-\lambda_{j}^{+}}, \forall k \in Z^{r},|i-j|+|k| \neq 0 .
$$

$$
\left|\langle\omega, k\rangle \sqrt{-1}+\lambda_{i}^{+}-\lambda_{j}^{+}\right| \geq \frac{\alpha_{+}}{|k| \tau^{\prime}}, \forall 0 \neq k \in Z^{r}, \forall i, j=1,2, \cdots n,
$$

where $\tau^{\prime}=3 \tau$, then $\left|p_{i j}^{k}\right| \leq\left(|k|^{\tau^{\prime}} / \alpha_{+}\right)\left|\tilde{q}_{i j}^{k}\right|$. Since $\tilde{Q}$ is analytic on $D_{\rho}$, we have $\left\|\tilde{Q}_{k}\right\| \leq\|\tilde{Q}\|_{D_{\rho}} e^{-|k| \rho}$. So

$$
\|P\|_{D_{\rho-s}} \leq \sum_{k \in Z^{r}}\left\|P_{k}\right\| e^{|k|(\rho-s)} \leq\left(\frac{1}{\delta}+\sum_{0 \neq k \in Z^{r}} \frac{|k|^{\tau^{\prime}} e^{-s|k|}}{\alpha_{+}}\right)\|\tilde{Q}\|_{D_{\rho}} \leq \frac{c}{\alpha_{+} s^{v}}\|Q\|_{D_{\rho}},
$$


where $v=\tau^{\prime}+r-1,0<s \leq \frac{1}{2} \rho, \delta=\min _{i \neq j}\left|\lambda_{i}^{+}-\lambda_{j}^{+}\right|$, and $c$ depends on $\tau^{\prime}, r, n$. Thus, If $\epsilon$ is sufficiently small that $\left\|(I+\epsilon P)^{-1}\right\|_{D_{\rho-s}} \leq 2$, then

$$
\left\|Q_{+}\right\|_{D_{\rho-s}} \leq \frac{2 c}{\alpha_{+} s^{v}}\|Q\|_{D_{\rho}}^{2} .
$$

B. Iteration step. Consider the following equations:

$$
\dot{x}_{m}=\left(A_{m}+\epsilon^{2^{m}} Q_{m}(t)\right) x_{m}, \quad m \geq 0
$$

where $A_{m}=\operatorname{diag}\left(\lambda_{1}^{m}, \lambda_{2}^{m}, \cdots, \lambda_{n}^{m}\right)$, and $Q_{m}(t)$ is an analytic quasiperiodic matrix on $D_{\rho_{m}}$ with the frequencies $\omega_{1}, \omega_{2}, \cdots, \omega_{r}$. Let

$$
A_{m+1}=\operatorname{diag}\left(\lambda_{1}^{m+1}, \lambda_{2}^{m+1}, \cdots, \lambda_{n}^{m+1}\right),
$$

where $\lambda_{i}^{m+1}=\lambda_{i}^{m}+\epsilon^{2^{m}} \bar{q}_{i i}^{m}, i=1,2, \cdots, n$, with $\bar{q}_{i i}^{m}$ being the average of $q_{i i}^{m}(t)$. Let $\tilde{Q}_{m}(t)=Q_{m}(t)-\epsilon^{2^{m}} \operatorname{diag}\left(\bar{q}_{11}^{m}, \bar{q}_{22}^{m}, \cdots, \bar{q}_{n n}^{m}\right)$.

If

$$
\left|\langle\omega, k\rangle \sqrt{-1}+\lambda_{i}^{m+1}-\lambda_{j}^{m+1}\right| \geq \frac{\alpha_{m}}{|k|^{3 \tau}},|k|+|i-j| \neq 0,
$$

then by the above discussions we have an analytic quasiperiodic matrix $P_{m}$ on $D_{\rho_{m}-s_{m}}$ with the frequencies $\omega_{1}, \omega_{2}, \cdots, \omega_{r}$, such that under the change of variables $x_{m}=\left(I+\epsilon^{2^{m}} P_{m}\right) x_{m+1}$, the equations (2.5) are changed to

$$
\dot{x}_{m+1}=\left(A_{m+1}+\epsilon^{2^{m+1}} Q_{m+1}(t)\right) x_{m+1} .
$$

Moreover, we have

$$
\left\|P_{m}\right\|_{D_{\rho_{m}-s_{m}}} \leq \frac{c}{\alpha_{m} s_{m}^{v}}\left\|Q_{m}\right\|_{D_{\rho_{m}}}^{2} .
$$

If $\epsilon$ is sufficiently small that $\left\|\left(I+\epsilon^{2^{m}} P_{m}\right)^{-1}\right\|_{D_{\rho_{m}-s_{m}}} \leq 2$, we have

$$
\left\|Q_{m+1}\right\|_{D_{\rho_{m}-s_{m}}} \leq \frac{2 c}{\alpha_{m} s_{m}^{v}}\left\|Q_{m}\right\|_{D_{\rho_{m}}}^{2}
$$

Now we prove this iteration is convergent. At the first step, let $A_{0}=A, Q_{0}(t)=$ $Q(t), \alpha_{0}=\alpha / 2, \rho_{0}=\rho, s_{0}=\rho / 4, D_{0}=D_{\rho_{0}}, F_{0}=\left(\epsilon\left\|Q_{0}\right\|_{D_{0}}\right) /\left(\alpha_{0} s_{0}^{v}\right)$. At the $m$ th step we choose $\alpha_{m}=\alpha_{0} /(1+m)^{2}, s_{m}=s_{m-1} / 2, \rho_{m+1}=\rho_{m}-s_{m}, D_{m}=$ $D_{\rho_{m}}, F_{m}=\left(\epsilon^{2^{m}}\left\|Q_{m}\right\|_{D_{m}}\right) /\left(\alpha_{m} s_{m}^{v}\right)$. If

$$
\left\|\left(I+\epsilon^{2^{m}} P_{m}\right)^{-1}\right\|_{D_{m+1}} \leq 2, \forall m \geq 0
$$

then by (2.8) we have $F_{m+1} \leq \bar{c} F_{m}^{2}$, where $\bar{c}=2^{v+1} c$. So $\bar{c} F_{m+1} \leq\left(\bar{c} F_{m}\right)^{2}$. If $\left|\bar{c} F_{0}\right|<\frac{1}{2}$, then $\bar{c} F_{m} \leq\left(\frac{1}{2}\right)^{2^{m}}$. By $(2.7)$ it follows that $\left\|\epsilon^{2^{m}} P_{m}\right\|_{D_{m+1}} \leq \bar{c} F_{m} \leq$ $\left(\frac{1}{2}\right)^{2^{m}}$. So, we have

$$
\left\|\left(I+\epsilon^{2^{m}} P_{m}\right)^{-1}\right\|_{D_{m+1}} \leq 1+\sum_{l=1}^{\infty}\left\|\epsilon^{2^{m}} P_{m}\right\|_{D_{m+1}}^{l} \leq 2 .
$$

Let $\epsilon_{1}>0$ be so small that $\bar{c} F_{0} \leq \frac{1}{2}$ for $\epsilon \in\left(0, \epsilon_{1}\right)$. Thus, for $\epsilon \in\left(0, \epsilon_{1}\right)$ satisfying all the small divisor conditions (2.6), all the above estimates hold.

Let $D_{*}=\bigcap_{m=0}^{\infty} D_{m}, P^{m}=\left(I+\epsilon^{2^{m}} P_{m}\right)\left(I+\epsilon^{2^{m-1}} P_{m-1}\right) \cdots\left(I+\epsilon P_{0}\right)$. Obviously, $D_{\frac{1}{2} \rho} \subset D_{*}$ and $P^{m}$ is convergent as $m \rightarrow \infty$ under the norm $\|\cdot\|_{D_{*}}$. Let $\Phi=$ $\lim _{m \rightarrow \infty} P^{m}$.

From the above discussion, it follows that $\left\|A_{m}-A_{m-1}\right\| \leq \epsilon^{2^{m}}\left\|Q_{m}\right\|_{D_{m}}$. So, $A_{m}$ is also convergent. Let $\lim _{m \rightarrow \infty} A_{m}=B$. Obviously, $\lim _{m \rightarrow \infty}\left\|\epsilon^{2^{m}} Q_{m}\right\|_{D_{\frac{1}{2} \rho}}=0$. 
Thus, if $\epsilon \in\left(0, \epsilon_{1}\right)$ satisfy the small divisor conditions (2.6) for all $m \geq 0$, then, under the change of variables $x=\Phi y$, the equations (1.1) become $\dot{y}=B y$. Moreover, $\Phi$ is an analytic quasiperiodic matrix on $D_{\frac{1}{2} \rho}$ with the frequencies $\omega_{1}, \omega_{2}, \cdots, \omega_{r}$.

To finish the proof of the theorem, it remains to prove that there exist $N_{1}, N_{2}, \cdots$, $N_{p}$ such that if $\left|\mu_{i}\right| \geq N_{i}(i=1,2, \cdots, p)$, then there exist $0<\epsilon_{0}<\epsilon_{1}$ and a nonempty Cantor subset $E \subset\left(0, \epsilon_{0}\right)$ such that for $\epsilon \in E$, the small divisor conditions (2.6) hold for all $m \geq 0$. Now we first prove that there exist $N_{1}, N_{2}, \cdots, N_{p}$ such that if $\left|\mu_{i}\right| \geq N_{i}, i=1,2, \cdots, p$, then for all $m \geq 0$ and $i \neq j$, there exist $l \in\left\{l_{1}, l_{2}, \cdots, l_{p}\right\}$ such that the $l$ th derivative of $\lambda_{i}^{m+1}-\lambda_{j}^{m+1}$ with respect to $\epsilon$ at $\epsilon=0$ does not vanish.

Suppose that $\lambda_{i}^{1}-\lambda_{j}^{1}=\mu_{1} \epsilon^{l_{1}}+o\left(\epsilon^{l_{1}}\right)$. Let $N_{1} \geq 0$ be an integer such that $2^{\bar{N}_{1}} \leq l_{1} \leq 2^{\bar{N}_{1}+1}$. Let

$$
\bar{M}_{i}^{1}=\left\|\left.\frac{d^{l_{1}-2^{i}} Q_{i}}{d \epsilon^{l_{1}-2^{i}}}\right|_{\epsilon=0}\right\|_{D_{\rho_{i}}}, i=1,2, \cdots, \bar{N}_{1} .
$$

Then $\bar{M}_{i}^{1}\left(i=1,2, \cdots, \bar{N}_{1}\right)$ only depends on $\tau, n, \alpha, \rho$, and $M_{0}, M_{1}, \cdots, M_{l_{1}-2^{i}}$. By the construction of the transformation, $\bar{M}_{i}^{1}$ should depend on all derivatives up to the $l_{1}-2^{i}$-th of $\lambda_{j_{1}}^{i}(\epsilon)-\lambda_{j_{2}}^{i}(\epsilon)$ with respect to $\epsilon$ at $\epsilon=0$. But these derivatives only depend on $M_{0}, M_{1}, \cdots, M_{l_{1}-2^{i}}$ and are independent of $\mu_{1}, \mu_{2}, \cdots, \mu_{p}$. Let

$$
N_{1}=\frac{\bar{M}_{1}^{1}+\bar{M}_{2}^{1}+\cdots+\bar{M}_{\bar{N}_{1}}^{1}}{l_{1} !}
$$

If $\left|\mu_{1}\right|>N_{1}$, we have at $\epsilon=0$

$$
\left|\frac{d^{l_{1}}\left(\lambda_{i}^{m+1}-\lambda_{j}^{m+1}\right)}{d \epsilon^{l_{1}}}\right| \geq l_{1} !\left|\mu_{1}\right|-\left(\bar{M}_{1}+\bar{M}_{2}+\cdots+\bar{M}_{\bar{N}_{1}}\right)>0 .
$$

Similarly, let $\bar{N}_{2} \geq \bar{N}_{1}$ be an integer such that $2^{\bar{N}_{2}} \leq l_{2}<2^{\bar{N}_{2}+1}$. Let

$$
\bar{M}_{i}^{2}=\left\|\left.\frac{d^{l_{2}-2^{i}} Q_{i}}{d \epsilon^{l_{2}-2^{i}}}\right|_{\epsilon=0}\right\|_{D_{\rho_{i}}}, i=1,2, \cdots, \bar{N}_{2} .
$$

Then $\bar{M}_{i}^{2}\left(i=1,2, \cdots, \bar{N}_{2}\right)$ only depends on $\tau, n, \alpha, \rho, M_{0}, M_{1}, \cdots, M_{l_{2}-2^{i}}$ and the $l$-th order derivatives of $\mu_{1} \epsilon^{l_{1}}+o\left(\epsilon^{l_{1}}\right)$ with respect to $\epsilon$, where $l \leq l_{2}-2^{i}$.

Let

$$
N_{2}=\frac{\bar{M}_{1}^{2}+\bar{M}_{2}^{2}+\cdots+\bar{M}_{\bar{N}_{2}}^{2}}{l_{2} !}
$$

If $\left|\mu_{2}\right|>N_{2}$, then, when $\lambda_{i}^{1}-\lambda_{j}^{1}=\mu_{2} \epsilon^{l_{2}}+o\left(\epsilon^{l_{2}}\right)$, for all $m \geq 0$, the $l_{2}$-th derivative of $\lambda_{i}^{m+1}-\lambda_{j}^{m+1}$ with respect to $\epsilon$ at $\epsilon=0$ does not vanish. From the above we see that $\bar{N}_{2}$ may depend on $\bar{N}_{1}$, but $\bar{N}_{1}$ is independent of $\bar{N}_{2}$. In the same way we can obtain $\bar{N}_{p}$, which depends on $\tau, n, \alpha, \rho, M_{0}, M_{1}, \cdots, M_{l_{p}-2}$ and the $l$-th derivatives of $\mu_{i} \epsilon^{l_{i}}+o\left(\epsilon^{l_{i}}\right)$ with respect to $\epsilon, i=1,2, \cdots, p-1$, where $l \leq l_{p}-2$. If $\left|\mu_{p}\right|>N_{p}$, then, when $\lambda_{i}^{1}-\lambda_{j}^{1}$ consists of $\mu_{p} \epsilon^{l_{p}}$, for all $m \geq 0$, the $l_{p}$-th derivative of $\lambda_{i}^{m+1}-\lambda_{j}^{m+1}$ with respect to $\epsilon$ at $\epsilon=0$ is not zero. It is easy to see that $N_{p}$ 
may depend on $N_{1}, N_{2}, \cdots, N_{p-1}$. Thus, if $\lambda_{i}^{1}-\lambda_{j}^{1}=\mu_{i} \epsilon^{l_{i}}+o\left(\epsilon^{l_{i}}\right)$, then

$$
\left.\frac{d^{l_{i}}\left(\lambda_{i}^{m+1}-\lambda_{j}^{m+1}\right)}{d \epsilon^{l_{i}}}\right|_{\epsilon=0} \neq 0, \forall m \geq 0
$$

From the above iteration we see that the first step can only be done for all $\epsilon \in E_{0} \subset\left(0, \epsilon_{0}\right)$, where $E_{0}$ is the set on which the small divisor conditions of the first step hold . Let $E_{m-1}$ be the set on which the small divisor conditions of the $m$-th step hold. Then the $m$-th step can only be done for $\epsilon \in E_{0} \cap E_{1} \cap \cdots \cap E_{m-1}$. Thus, the above iteration can only be convegent on the set $E=\bigcap_{m=0}^{\infty} E_{m}$. Since the small divisor conditions hold on a Cantor subset, in all the iteration steps the differentiations with respect to $\epsilon$ are understood in Whitney's sense [5]. By the Whitney extension theorem in [5], all differentiable functions on a closed set in Whitney's sense can be extended to the usual differentiable function on $(-\infty,+\infty)$ and the differentiation in Whitney's sense can be treated as the usual differentiation. Thus all the functions in the iteration step can be regarded as regular differentiable functions on $(-\infty,+\infty)$, and so all the estimates can be obtained without any difficulty . However, the iteration makes sense only for $\epsilon \in E$.

Now we prove $E$ is a nonempty set. For this we prove that for most sufficiently small $\epsilon$, the small divisor conditions (2.6) hold for all $m \geq 0$. Let $\lambda_{i}^{m+1}-\lambda_{j}^{m+1}=$ $\mu_{l} \epsilon^{l}+o\left(\epsilon^{l}\right)$, where $l \leq l_{p}$. It is easy to see that the term $\mu_{l} \epsilon^{l}$ is unvariant when $m \geq \bar{N}_{p}$. So there exists a sufficiently small $\epsilon_{0}>0$ such that if $|\epsilon| \leq \epsilon_{0}$, then $\left|\lambda_{i}^{m+1}-\lambda_{j}^{m+1}\right| \leq 2\left|\mu_{l}\right| \epsilon^{l}$ and $\left|\frac{d\left(\lambda_{i}^{m+1}-\lambda_{j}^{m+1}\right)}{d \epsilon}\right| \geq \frac{1}{2}\left|\mu_{l}\right| \epsilon^{l-1}$ for all $m \geq 0$.

Let $f(\epsilon)=\langle\omega, k\rangle+\lambda_{i}^{m+1}-\lambda_{j}^{m+1}, i \neq j$, and

$$
O_{i j m}^{k}=\left\{\epsilon \in\left(0, \epsilon_{0}\right) \quad|\quad| f(\epsilon) \mid<\frac{\alpha_{m}}{|k|^{3 \tau}}\right\} .
$$

Since $\lambda_{i}^{0}-\lambda_{j}^{0} \neq 0, \forall i \neq j$, we choose $\epsilon_{0}$ so small that if $|\epsilon| \leq \epsilon_{0},\left|\lambda_{i}^{m+1}-\lambda_{j}^{m+1}\right| \geq$ $\bar{\alpha}>0$ holds for all $i \neq j$ and $m \geq 1$. So we only consider $k \neq 0$. Since

$$
\left|\langle\omega, k\rangle+\lambda_{i}^{m+1}-\lambda_{j}^{m+1}\right| \geq\left|\langle\omega, k\rangle \sqrt{-1}+\lambda_{i}^{0}-\lambda_{j}^{0}\right|-2 \mu_{l} \epsilon^{l},
$$

by the nonresonance conditions of the theorem, if $1 /|k|^{\tau}>4 \mu_{l} \epsilon^{l} / \alpha_{0}$, then $|f(\epsilon)| \geq$ $\alpha_{0} / 2|k|^{\tau}>\alpha_{m} /|k|^{3 \tau}$.

Suppose $\alpha_{0} / 4 \mu_{l}|k|^{\tau}<\epsilon^{l} \leq \epsilon_{0}$. Since

$$
\left|\frac{d f(\epsilon)}{d \epsilon}\right| \geq \frac{1}{2}\left|\mu_{l}\right| \epsilon^{l-1}
$$

by the differentiation mean value theorem, we have

$$
\operatorname{meas}\left(O_{i j m}^{k}\right) \leq \frac{2 \alpha_{m}}{|k|^{3 \tau}} \frac{2}{\left|\mu_{l}\right| \epsilon^{l-1}} \leq \frac{\epsilon_{0}^{l+1}}{|k|^{\tau}(m+1)^{2}} \frac{4\left|\mu_{l}\right|}{\alpha_{0}} \leq \frac{8\left|\mu_{l}\right| \epsilon_{0}^{l+1}}{\alpha(m+1)^{2}|k|^{\tau}} .
$$

So

$$
\operatorname{meas}\left(\bigcup_{i \neq j} \bigcup_{0 \neq k \in Z^{r}} O_{i j m}^{k}\right) \leq \frac{8 n^{2} \max _{l}\left|\mu_{l}\right| \epsilon_{0}^{2}}{\alpha} \sum_{m=0}^{\infty} \frac{1}{(m+1)^{2}} \sum_{0 \neq k \in Z^{r}} \frac{1}{|k|^{\tau}}=c \epsilon_{0}^{2},
$$

where $c$ depends on $n, \tau, \alpha$ and $\mu_{l}$. Let $E$ be the subset of $\left(0, \epsilon_{0}\right)$ where the small divisor conditions (2.6) hold for all $m \geq 0$. Then $E=\left(0, \epsilon_{0}\right)-\bigcup_{m, i, j, k} O_{i j m}^{k}$. Thus $\operatorname{meas}(E) \geq \epsilon_{0}-c \epsilon_{0}^{2}=\epsilon_{0}\left(1-c \epsilon_{0}\right)$. If $\epsilon_{0}$ is so small that $1-c \epsilon_{0}>0$. then $E$ is a nonempty set with positive Lebesgue measure. Noticing that $\left\{\frac{k}{|k|} \mid 0 \neq k \in Z^{r}\right\}$ is dense on the unit ball of $R^{r}$, we conclude that $E$ is a Cantor set. 


\section{ACKNOWLEDGEMENT}

We would like to thank the referee for much helpful advice which we used in our revision.

\section{REFERENCES}

[1] N. N. Bogoljubov, J. A. Mitropoliski and A. M. Samoilenko, Methods of Accelerated Convergence in Nonlinear Mechanics, Springer-Veriage, New York (1976). MR 53:1156

[2] Angel Jorba and Carles Simó, On the Reducibility of Linear Differential Equations with Quasiperiodic Coefficients, J. Diff. Equa. 98 (1992), 111-124. MR 94f:34024

[3] V. I. Arnold, Small denominators and problems of stability of motion in classical and celestial mechanics, Russian Math. Surveys 18 (1963), no. 6, 85-191. MR 30:943

[4] Pöschel, J., On elliptic lower dimensional tori in Hamiltonian systems, Math. Z. 202 (1989), 559-608. MR 91a:58065

[5] Whitney, H., Analytical extensions of differentiable functions defined in closed sets, Trans. A.M.S. 36 (1934), 63-89.

Department of Mathematics and Mechanics, Southeast University, Nanjing 210096, People's Republic of China

E-mail address: xujun@seu.edu.cn

Department of Mathematics, Nanjing University, Nanjing 210093, People's Republic OF ChINA 